\title{
Research on Harmonic Characteristic of Converter
}

\author{
Zhong Binbin \\ School of Electrical and Electronic Engineering \\ Huazhong University of Science and Technology \\ WUHAN, CHINA \\ zhongbinbin@hust.edu.cn
}

Li Yan

School of Electrical and Electronic Engineering Huazhong University of Science and Technology WUHAN, CHINA

\section{Chen Wei}

School of Electrical and Electronic Engineering Huazhong University of Science and Technology WUHAN, CHINA

\author{
Zhang Yongfang \\ School of Electrical and Electronic Engineering \\ Huazhong University of Science and Technology \\ WUHAN, CHINA \\ $\mathrm{Hu}$ Baolin \\ School of Electrical and Electronic Engineering \\ Huazhong University of Science and Technology \\ WUHAN, CHINA
}

\begin{abstract}
Power electronic Converter is an electrical components connecting AC / DC networks. It is important to study the AC / DC sides harmonic characteristics. Based on the improved switching function which is processed with multiple states, the paper gets the modulation waveform, which is modulated by the improved switching function and the voltage / current waveforms. And the harmonic characteristics of converter's both sides by the Fourier series expansion is derived. Then the article quantificationally studys the converter harmonic characteristics through three aspects, that is " the DC side harmonics characteristics under the AC side mixed system background harmonics condition; the AC side harmonics characteristics under DC side mixed system background harmonics condition; and the AC side current harmonics self-coupling under AC side voltage mixed system background harmonics condition". The method has broad applicability in converter harmonics anslysis with symmetric system background harmonics on AC side. Simulink time-domain simulation results proves the accuracy of this method lastly.
\end{abstract}

Keywords-Converter; Switching Function; Modulation Theory; Harmonic Coupling; Harmonic Characteristics

\section{INTRODUCTION}

With wide application of power electronic devices in recent years, the power grid has a proliferation of harmonic source, such as HVDC systems, electric vehicle charging stations, variable-frequency drives.[1-2] Converters are the basic blocks of the above systems. Studies on converter harmonic characteristics, is not only the basis of above system,but also the foundation of the cluster converters impacting power grid.

Harmonic frequency domain methods generally get through the analysis of the working situations of switching devices, then use Fourier harmonic expression[3-5], but the condition switching devices of trigger synchronous should be fulfilled.Reference[6-7] expresses the ideal working situation of switching devices as switching functions and Fourier series expansion, and the converter device can be equivalent to a linear function with physical meaning and simple expression; but it exists big error without considerding the influence of the rectifier commutation overlap angle. In engineer practice, considering the firing angle modulated by cosine signal, reference [8-9] utilizes the pulse width modulation and pulse position modulation to analyze the frequency spectrum, and applies the transfer function to analyze both sides of inverter harmonics distribution. The method is convenient and accurate, but neglects the case existing background harmonics on both sides of inverter.

Based on the improved switching function with multiple state processing,this paper gets modulation waveform of switching function and the voltage/ current waveform. Then gets the harmonic characteristic of converter's both sides by the Fourier series expansion.The article quantificationally studys on"the DC side harmonics characteristics under $\mathrm{AC}$ side mixed background harmonics; the AC side harmonics characteristics under DC side mixed background harmonics; and the AC side current harmonics self-coupling under $\mathrm{AC}$ side voltage mixed background harmonics". The method has broad applicability with symmetric background harmonics on AC side. Simulink simulation proves the accuracy of this method.

\section{IMPROVED SWITCHING FUNCTION}

\section{A. Votlage Switching Function on DC side}

The votlage switching function on DC side can be described by thyristors four consecutive working situations $(0, \quad 0.5,1$ and 0.5), namely, cut-off, conduction commutation process, conduction and cut-off commutation process. The switching function on DC side of each thyristor is as given in (1) 


$$
\begin{gathered}
f(m)=\left\{\begin{array}{l}
0.5, a+\frac{\pi}{6} \leq w t \leq a+\frac{\pi}{6}+u \\
1, a+\frac{\pi}{6}+u \leq w t \leq a+\frac{5 \pi}{6} \\
0.5, a+\frac{5 \pi}{6} \leq w t \leq a+\frac{5 \pi}{6}+u
\end{array}\right. \\
a=a_{0}+\frac{(m-1) \pi}{3} \\
u=\cos ^{-1}\left(\cos a_{0}-\frac{2 w L_{s} I_{d 0}}{\sqrt{3} U_{1}}\right)-a_{0}, \text { it was agreed }
\end{gathered}
$$

that the harmonic emf amplitudeon $\mathrm{AC}$ side is so small that no affecting the normal working of switching devices [10-11]. $\mathrm{a} 0$ is the firing angle, $\mathrm{u}$ is the commutation overlap angle, Ls is the converter transformer short-circuit reactance, $\mathrm{U} 1$ is fundamental phase voltage amplitude. The switching function on DC side of each thyristor is shown in Fig .1.

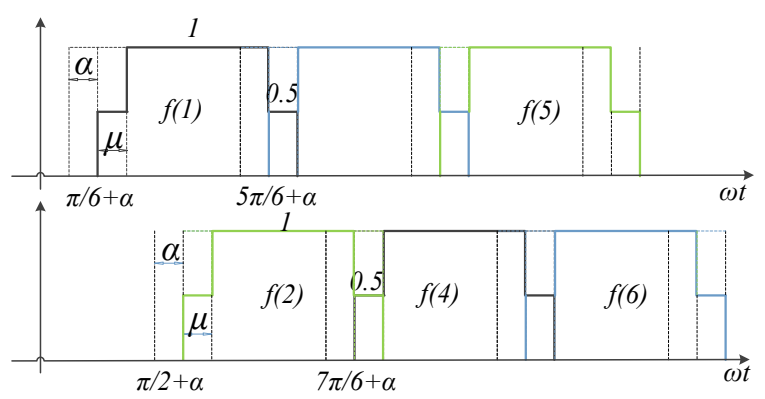

Figure 1. votlage switching function on DC side

The switch function Fourier series expansion of (1) is $f(m)=\frac{1}{3}+\sum_{k=1,2,3 \ldots}^{\infty} \frac{2}{k \pi} \sin \frac{k \pi}{3} \cos \frac{k u}{2} \cos k\left(w t-\frac{\pi}{2}-a_{0}-\frac{u}{2}\right)$

The DC side voltage switching function of A, B, C phase is as given in (3).

$$
\left\{\begin{array}{l}
S_{U_{a}}=f(1)-f(4) \\
S_{U_{b}}=f(3)-f(6) \\
S_{U_{c}}=f(5)-f(2)
\end{array}\right.
$$

Take A phase for example, its votlage switch function Fourier series expansion is as given in (4).

$$
\begin{aligned}
S_{U_{a}} & =f(1)-f(4) \\
& =\sum_{k=1,2,3 \ldots}^{\infty} \frac{4}{k \pi} \sin \frac{k \pi}{3} \sin \frac{k \pi}{2} \cos \frac{k u}{2} \sin k\left(w t-a_{0}-\frac{u}{2}\right)
\end{aligned}
$$

\section{B. Current Switching Function on AC side}

Current switching function on AC side can also get throngh thyristors cut-off, conduction commutation process, conduction and cut-off commutation process four four continuous working status, namely 0 , the linear function with slope $1 / \mathrm{u}, 1$, and the linear function with slope $-1 / \mathrm{u}$. The switching function on AC side of each thyristor is as given in (5) and shown in Fig .2.

$$
g(m)=\left\{\begin{aligned}
\frac{1}{u}\left(w t-\frac{\pi}{6}-a\right), a+\frac{\pi}{6} & \leq w t \leq a+\frac{\pi}{6}+u \\
1, a+\frac{\pi}{6}+u \leq w t & \leq a+\frac{5 \pi}{6} \\
-\frac{1}{u}\left(w t-\frac{5 \pi}{6}-a-u\right), a+\frac{5 \pi}{6} & \leq w t \leq a+\frac{5 \pi}{6}+u
\end{aligned}\right.
$$

The switch function Fourier series expansion of (5) is $g(m)=\frac{1}{3}+\sum_{k=1,2,3 \ldots}^{\infty} \frac{4}{k^{2} u \pi} \sin \frac{k \pi}{3} \sin \frac{k u}{2} \cos k\left(w t-\frac{\pi}{2}-a_{0}-\frac{u}{2}\right)(6)$

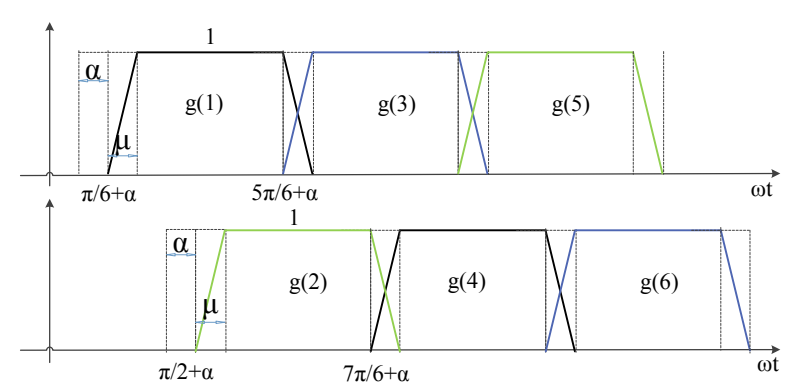

Figure 2. current switching function on AC side

Take A phase for example, its current switch function Fourier series expansion is as given in (7).

$$
\begin{aligned}
S_{i_{a}} & =g(1)-g(4)=\frac{2 \sqrt{3}}{\pi} \frac{\sin \frac{u}{2}}{\frac{u}{2}} \sin \left(w t-a_{0}-\frac{u}{2}\right)+ \\
& \frac{2 \sqrt{3}}{\pi} \sum_{k=1,2,3 \ldots}^{\infty} \frac{(-1)^{k}}{(6 k \pm 1)} \sin \left[(6 k \pm 1)\left(w t-a_{0}-\frac{u}{2}\right)\right] \frac{\sin \frac{k u}{2}}{\frac{k u}{2}}
\end{aligned}
$$

\section{HARMONIC CHARACTERISTIC OF CONVERTER}

\section{A. Harmonic Characteristic on DC side}

Considering the general condition, the bus of converter AC side contains symmetrical system background harmonic votlage, the subscript $\mathrm{m}$ and $\mathrm{s}$ respectively harmonic voltage frequency and phase sequence. Threephase voltage symmetrical component is expressed with symmetrical component shown in (8).

$$
\left\{\begin{array}{c}
u_{a}=\sum_{s=-1,0,1} \sum_{m=1,2,3 \cdots}^{\infty} U_{\mathrm{sm}} \sin \left(m w t+a_{s m}\right) \\
u_{b}=\sum_{s=-1,0,1} \sum_{m=1,2,3 \cdots}^{\infty} U_{\mathrm{sm}} \sin \left(m w t+a_{s m}-s \frac{2 \pi}{3}\right) \\
u_{c}=\sum_{s=-1,0,1} \sum_{m=1,2,3 \cdots}^{\infty} U_{\mathrm{sm}} \sin \left(m w t+a_{s m}+s \frac{2 \pi}{3}\right)
\end{array}\right.
$$

The converter DC side voltage can be expressed by ac three-phase voltage and voltage switching function as shown in (9).

$$
U_{d}=S_{U_{a}} u_{a}+S_{U_{b}} u_{b}+S_{U_{c}} u_{c}=U_{d}{ }^{\prime}+U_{d}{ }^{\prime \prime}
$$

This article will analyze the DC side harmonic influence dividing into ac voltage fundamental component and harmonic component respectively.

1) The Influence of Fundamental Voltage 
According to (2),(3),(4)and (9), which $\mathrm{m}=1$, DC side voltage harmonic characteristics under the condition of ac three-phase fundamental voltage is shown in (10).
It is shown that DC side votlage contains $\mathrm{dc}$ component and characteristic harmonic order $6 k_{1}$ $(k=1,2,3 \ldots)$.

$$
\begin{aligned}
U_{d}{ }^{\prime}= & \frac{3 \sqrt{3} U_{1}}{\pi} \cos \frac{u}{2} \cos \left(\frac{u}{2}+a_{0}-a_{11}\right)+ \\
& \frac{3 \sqrt{3} U_{1}}{\pi} \sum_{k=1,2,3 \ldots}^{\infty}\left\{\begin{array}{l}
\frac{(-1)^{k}}{6 k+1} \cos \left[6 k w t-(6 k+1)\left(a_{0}+\frac{u}{2}\right)-a_{11}\right] \bullet \cos \frac{(6 k+1) u}{2} \\
\left.-\frac{(-1)^{k}}{6 k-1} \cos \left[6 k w t-(6 k-1)\left(a_{0}+\frac{u}{2}\right)+a_{11}\right] \bullet \cos \frac{(6 k-1) u}{2}\right]
\end{array}\right\} \\
U_{d}{ }^{\prime}= & \frac{3 \sqrt{3} U_{m}}{\pi} \sum_{s=-1,1} \sum_{m=2,3,4 \ldots k=1,2,3 \ldots}^{\infty}\left\{\begin{array}{l}
\frac{(-1)^{k}}{6 k+1} \cos \left\{[m-s(6 k+1)] w t+s(6 k+1)\left(a_{0}+\frac{u}{2}\right)+a_{s m}\right\} \bullet \cos \frac{(6 k+1) u}{2} \\
-\frac{(-1)^{k}}{6 k-1} \cos \left\{[m+s(6 k-1)] w t-s(6 k-1)\left(a_{0}+\frac{u}{2}\right)+a_{s m}\right\} \bullet \cos \frac{(6 k-1) u}{2}
\end{array}\right.
\end{aligned}
$$

\section{2) The Influence of Harmonic Voltage}

Similarly, DC side voltage harmonic characteristics under the condition of ac three-phase harmonic voltage is shown in (11), which $m>1$.

It can be odserved that the positive sequence $m_{1}$ thdegree harmonic voltage on AC side will produces harmonics of orders $\left|m_{1} \pm 6 k_{1}-1\right|$ on DC side. The negative sequence $m_{1}$ th-degree harmonic voltage on AC side will produces harmonics of orders $\left|m_{1} \pm 6 k_{1}-1\right|$ on DC side.The amplitude of DC side harmonic voltage is related to the AC side harmonic voltage, moreover, zero sequence ac harmonic voltage will have no influence on the converter DC side.

\section{B. Harmonic Characteristic on AC side}

Considering the general condition similarly, the DC side current contains dc component and harmonic components shown in (12).

$$
I_{d}=\frac{U_{d}}{R+j w L}=I_{d 0}+i_{d}
$$

The article study the influence of DC side dc currernt and harmonic curren on AC side current respectively.

$$
i_{a}=S_{i_{a}} I_{d 0}+S_{i_{a}} i_{d}=i_{a}{ }^{\prime}+i_{a}{ }^{\prime \prime}
$$

The AC side current harmonic characteristic will be modulated by current switching function and DC DC side dc current obtained by (14) is shown in (16). It is shown that $\mathrm{AC}$ side current contains fundamental component and characteristic harmonic order $6 k_{2} \sharp \quad(k=1,2,3 \ldots)$, and the amplitude of harmonics is $1 /\left(6 k_{2} \pm 1\right)$ of the fundamental component approximatively.

$$
I_{d 0}=\frac{3 \sqrt{3} U_{1}}{\pi R} \cos \frac{\mu}{2} \cos \left(\frac{\mu}{2}+\alpha_{0}-a_{11}\right)
$$

\section{2) The Influence of Harmonic Current}

To analyze the influence of DC side harmonic current on AC side current detailedly, this article sets DC side harmonic current given in (15), which contains each harmonic.

$$
i_{d}=\sum_{m=1,2,3 \ldots}^{\infty} I_{d m} \cos \left(m w t+\varphi_{d m}\right)
$$

Through (2) modulated by current switching function, $m_{2}$ th-degree harmonic current on DC side will produces harmonics of orders $\mid m_{2} \pm\left(6 k_{2} \pm\right.$ ) $\mid$ on AC side, which is shown in (17). Seriously when $m_{2}=1,5,7, \ldots$, it will be coupling dc biasing current [12].

\section{1) The Influence of DC Current}

$$
\begin{aligned}
i_{a}{ }^{\prime} & =S_{i_{a}} I_{d}=[g(1)-g(4)] I_{d 0} \\
& =\frac{2 \sqrt{3} I_{d 0}}{\pi} \frac{\sin \frac{u}{2}}{\frac{u}{2}} \sin \left(w t-a_{0}-\frac{u}{2}\right)+\frac{2 \sqrt{3} I_{d 0}}{\pi} \sum_{k=1,2,3 \ldots}^{\infty} \frac{(-1)^{k}}{6 k \pm 1} \sin \left[(6 k \pm 1)\left(w t-a_{0}-\frac{u}{2}\right)\right] \frac{\sin \frac{k u}{2}}{\frac{k u}{2}} \\
i_{a}{ }^{\prime \prime} & =i_{d} S_{i_{a}}=\frac{2 \sqrt{3}}{\pi} \frac{\sin \frac{u}{2}}{\frac{u}{2}} \sum_{m=1,2,3 \ldots}^{\infty} I_{d m}\left\{\cos \left[(m-1) w t+\varphi_{d m}+\left(a_{0}+\frac{u}{2}\right)\right]-\cos \left[(m+1) w t+\varphi_{d m}-\left(a_{0}+\frac{u}{2}\right)\right]\right\}+ \\
& \frac{2 \sqrt{3}}{\pi} I_{d m} \sum_{m=1,2,3 \ldots k=1,2,3 \ldots}^{\infty} \frac{(-1)^{k}}{6 k \pm 1}\left\{\cos \left[(m-6 k \mathrm{ml}) w t+\varphi_{d m}+(6 k \pm 1)\left(a_{0}+\frac{u}{2}\right)\right]\right\}
\end{aligned}
$$

\section{3) Self-coupling on AC side}

Based on the above analysis, the paper studies quadratic coupled harmonic between $\mathrm{AC}$ side harmonic voltage and curren.
Fundamental three-phase voltage on AC side produced $\mathrm{dc}$ component and characteristic harmonic order $6 k$ on DC side. Then the corresponding dc current will produces the fundamental component and characteristic harmonic order $6 k_{2} \#$ on AC side through modulated by current switching 
function, and the $6 k$ th-degree harmonic current will produces characteristic harmonic order $6 k_{2} \sharp$ on AC side. That is to say, there exists characteristic harmonic only both $\mathrm{AC} / \mathrm{DC}$ side where ideal ac three-phase voltage.

The AC side $\mathrm{m}$ th-degree harmonic voltage produced noncharacteristic harmonic order $|m \pm(6 k \pm 1)|$ on DC side. Then the corresponding $|m \neq(6 k \pm 1)|$ th-degree harmonic current will produces noncharacteristic harmonic order $|m \pm 6 k|$ and $|m \pm 6 k \pm 2|$ on AC side. Its each harmonic amplitude and phase can be obtained through (11) and (17). Because after two switching function modulation coupling, the amplitude of characteristic harmonic current by selfcoupling is relatively small.

\section{SIMULATION}

This section establishes three-phase rectifier circuit in Simulink, sets the phase-phase voltage RMS $V=400 \mathrm{~V}, \mathrm{AC}$ side inductance $L_{s}=0.2 \mathrm{mH}$; DC side load resistance $R=5 \Omega$, load inductance $L=0.5 \mathrm{H}$; and considers that ac three-phase voltage contains second harmonic voltage whose amplitude is $10 \%$ of the fundamental component.

According above analysis, there will be characteristic harmonic of orders $6,12,18 \ldots$ and noncharacteristic harmonic of orders $1,3,5,7 \ldots$ on $\mathrm{DC}$ side. On AC side, there will be characteristic harmonic of orders $5,7,11,13 \ldots$ and noncharacteristic harmonic of orders $0,2,4,6 \ldots$. The Fig .3 DC side voltage spectrum analysis and Fig .4 AC side current spectrum analysis indicates that the analysis method of harmonic characteristics coupled has higher accuracy.

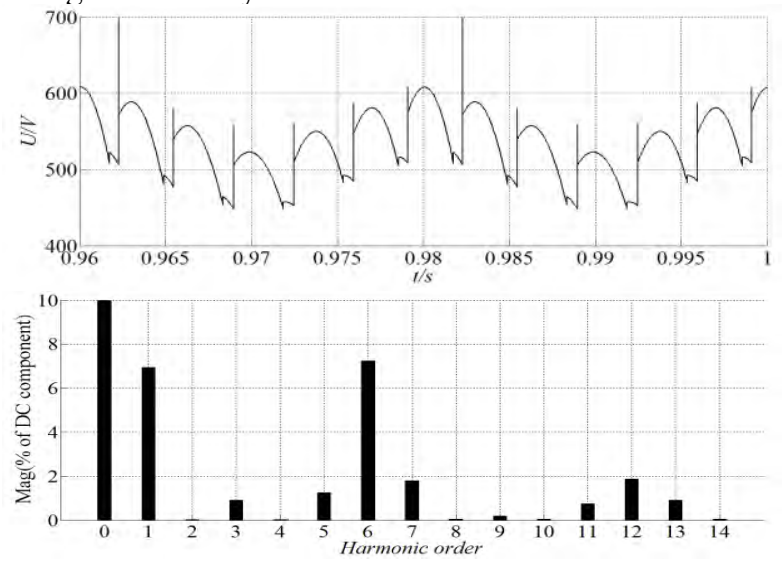

Figure 3. DC side votlage Spectrum analysis

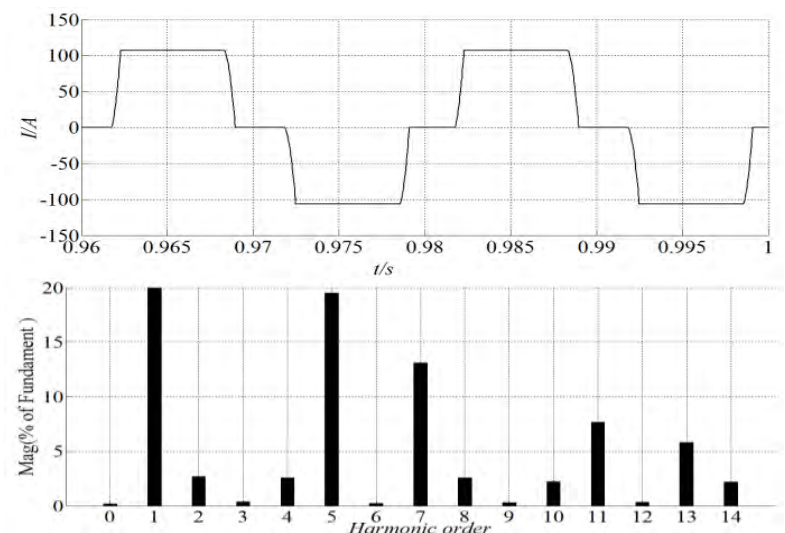

Figure 4. AC side current Spectrum analysis

\section{CONCLUSION}

This paper derives the multiple state switching function in detail, and gets modulation waveform of switching function and the voltage/ current waveform. Then gets the harmonic characteristic of converter's both sides by the Fourier series expansion.The article quantificationally studys on"the DC side harmonics characteristics under AC side mixed background harmonics; the AC side harmonics characteristics under DC side mixed background harmonics; and the AC side current harmonics selfcoupling under AC side voltage mixed background harmonics". The method has broad applicability with symmetric background harmonics on AC side. Simulink simulation proves the accuracy of this method finally.

\section{ACKNOWLEDGMENT}

The National Basic Research Program of China (973 Program) (2012CB215100-G); Independent Innovation Research Foundation of HUST (2012QN200).

\section{REFERENCES}

[1] PANG Hui, WEN Jialiang, HE Zhiyuan, et al. Unbalancing Voltage of High Power Series Connected IGBT Valve[J]. Proceedings of the CSEE, 2011, 31 (21) : 1 8.

[2] Marouchos . C. , Darwish M. K, El-Habrouk M. New mathematical model for analysing three-phase controlled rectifier using switching functions $[\mathrm{J}]$. Power Electronics IET, 2010, 3(1) : 95 110.

[3] Zheng Lianqing, Wu Ping, Li Kun. Modeling Approaches of Harmonic Sources in Power System[J] . Power System Technology, 2010, 34 (8) : 46-50.

[4] R Burch, G Chang, R Dwyer, et al. Characteristics and Modeling of Harmonic Sources-power Electronic Devices[J]. IEEE Transactions on Power Delivery, 2001, 16 (4) : 791- 800 .

[5] De Souza LFW, Watanabe E . H . Eliminating Gibbs Phenomenon From Switching Functions for Power Electronics Circuit Analysis $[\mathrm{J}]$. IEEE Transactions on Power Delivery, 2009, 24(2) : 970 971.

[6] Byoung-kuk Lee. A simplified function simulation model for three-phase voltage-source inverter using switching function concept[J]. IEEE Transactions on Industrial Electronics, 2001, 48 (2) : 309-317.

[7] LI Yuneng. Harmonic analysis of applying switching function to currernt converter[J] . Power System Technology, 2000 , 24(6) : $18 \sim 20$.

[8] Jos Arrillage, Nevile R. Watson. Power Sytem Haromics (second Edition) $[\mathrm{M}]$. Chichester : John Wiley \& Sons, Ltd, 2004 : 143-187.

[9] Liu Hong, Jiao Lianwei. Harmonic Analysis Using Transfer Function on the Converter[J] . Transactions of China Electrotechnical Society, 2005, 34 (9) : 108-113.

[10] XU Zheng. Analysis on AC/DC power system dynamic behavior [M]. Beijing : China Machine Press, 2005.

[11] Li Qionglin, Liu Huijin, Liu Yun . Uniform Modulation Modeling of Three-phase Converterfor the Analysis of Harmonic /Interharmonic[J] . High Voltage Engineering, 2008, 34 (4) : 718 722.

[12] MA Weimin . DC Biasing Current in Converter Transformer[J]. High Voltage Engineering, 2004,30 (11) : $48 \sim 49$. 